\title{
MiR-370 promotes apoptosis in colon cancer by directly targeting MDM4
}

\author{
XIAOGANG SHEN ${ }^{1}$, XIAOFEI ZUO ${ }^{1}$, WENJIN ZHANG $^{2}$, YIFENG BAI $^{3}$, XIANPENG QIN $^{1}$ and NENGYI HOU $^{1}$ \\ ${ }^{1}$ Department of Gastrointestinal Surgery, Sichuan Academy of Medical Sciences and Sichuan Provincial People's Hospital, \\ Chengdu, Sichuan 610041; ${ }^{2}$ Department of Clinical Medicine, Chengdu Medical College, Chengdu, Sichuan 610083; \\ ${ }^{3}$ Department of Oncology, Sichuan Academy of Medical Sciences and Sichuan Provincial People's Hospital, \\ Chengdu, Sichuan 610041, P.R. China
}

Received June 3, 2017; Accepted October 16, 2017

DOI: $10.3892 / \mathrm{ol} .2017 .7524$

\begin{abstract}
MicroRNA (miR)-370 functions as a tumor suppressor or promoter in several cancers. However, the expression and biological role of miR-370 in colon cancer remains undefined. In the present study, miR-370 expression in both normal and malignant colon tissues was quantified by quantitative polymerase chain reaction. An in vitro cell viability and apoptosis assay and an in vitro xenograft tumor model were employed to investigate the role of miR-370 on colon cancer growth. Furthermore, the potential direct target of miR-370 was identified using a luciferase assay. Our results demonstrate that down-regulation of miR-370 expression occurs in malignant tissues and miR-370 expression is inversely correlated with tumor grade. Moreover, we determined that miR-370 functions as a tumor suppressor in colon cancer by inhibiting cell proliferation and promoting cell apoptosis. In addition, overexpression of miR-370 impairs xenograft tumor growth in nude mice. Mechanistically, mouse double minute 4 (MDM4) was demonstrated to be a potential direct target of miR-370, inducing apoptosis in colon cancer. Collectively, these findings suggest that upregulation of miR-370 may impair colon tumor growth by directly targeting MDM4. These findings provide a new direction for the diagnosis and treatment of colon cancer.
\end{abstract}

\section{Introduction}

Colon cancer is the third most commonly diagnosed malignancy and the fourth leading cause of cancer mortality worldwide (1). Its late tumor presentation and rapid progression contribute to the high mortality rate (1). Colorectal neoplasia appears to be influenced by environmental factors and an accumulation of mutations that result in uncontrolled

Correspondence to: Dr Nengyi Hou, Department of Gastrointestinal Surgery, Sichuan Academy of Medical Sciences and Sichuan Provincial People's Hospital, 32 West Second Section First Ring Road, Chengdu, Sichuan 610041, P.R. China

E-mail: hounengyi11@126.com

Key words: colon cancer, miR-370, mouse double minute 4, apoptosis proliferation and angiogenesis, inhibition of apoptosis, and immune escape (2-4). Therefore, an improved understanding of the molecular mechanisms of colon tumorigenesis and progression may provide more options for treatment and improved prognosis of colorectal cancer patients.

It is known that less than $2 \%$ of the mammalian genome represents protein-coding genes and over $90 \%$ represents noncoding RNAs (ncRNA), which are transcribed but not translated into proteins $(5,6)$. MicroRNAs (miRNAs/miRs) belong to a class of small (typically 18-25 nucleotides), single-stranded ncRNAs that regulate post-transcriptional gene expression by base-pairing with complementary sequences on mRNA and inhibiting protein translation $(5,6)$. Recently, growing evidence has shown that miRNAs may be involved in the pathogenesis of several cancers, providing new insight into the biology of cancer (7-10). A previous study has indicated that miR-370 functions as a tumor suppressor in laryngeal squamous cell carcinoma (LSCC) through downregulation of FoxM1 (11). In ovarian cancer, miR-370 was down-regulated by hypermethylation and overexpression of miR-370 suppressed ovarian cancer cell viability, reduced colony formation, and enhanced ovarian cancer cell chemosensitivity to cisplatin (cDDP) by directly targeting Endoglin (ENG) (12). However, results by Wu et al demonstrated that miR-370 plays an important role in the proliferation of human prostate cancer cells, by directly suppressing the tumor suppressor FOXO1, which has been associated with key cellular functions including cell growth, differentiation, apoptosis, and angiogenesis (13).

The biological role of miR-370 in colon cancer and the underlying molecular mechanism remains undefined. In the present study, we studied the expression pattern of miR-370 and investigated its function in human colon cancer. Moreover, the regulation of colon cancer apoptosis by miR-370 was also examined in order to clarify the underlying mechanism of action. Our findings will provide a novel target in the diagnosis and treatment of colon cancer.

\section{Materials and methods}

Clinical sample. Human normal colon tissues and colonic tissues were obtained with informed consent under a general waiver by the Academic Medical Center Institutional Review Board for 
the proper secondary use of human material and were obtained from the People's Hospital of Sichuan from October 2016 to December 2016. Experiments described were approved by the Ethics Committee of Sichuan Academy of Medical Sciences and Sichuan Provincial People's Hospital (Chengdu, China). The tumor grade were identified according to clinical diagnosis.

Cell culture and treatment. Human colon cancer cell HT29, DLD-1, SW480, LoVo, HCT116 and SW620 were obtained from the American Type Culture Collection (Manassas, VA, USA). The human colon epithelial cell HCoEpiC was purchased from Shanghai Guandao Biotechnology. The cells were cultured in DMEM medium containing $10 \%$ fetal bovine serum (both from Gibco; Thermo Fisher Scientific, Inc., Waltham, MA, USA) and $1 \%$ penicillin and streptomycin at $37^{\circ} \mathrm{C}$ in $5 \% \mathrm{CO}_{2}$ incubator. The plasmid-based miR-370 expression system and miR-NC were transfected into cells using FuGENE HP ${ }^{\circledR}$ (Roche Diagnostics, Basel, Switzerland). The mouse double minute 4 (MDM4) specific inhibitor NSC207895 was purchased from Selleck Bio (MA, USA) and used to treat LoVo cells that transfected with miR-NC or miR-370.

Cell Counting kit-8 (CCK-8) kit. For cell viability detection, cells were seeded at 800 cells per well in 96 -well plates. Then, a CCK-8 assay (Beyotime Institute of Biotechnology, Shanghai, China) was used and absorbance was measured at $450 \mathrm{~nm}$ for each well at different time points using a micro-plate reader (Thermo Fisher Scientific, Inc.). The same experiments were performed four times.

Quantitative real-time polymerase chain reaction ( $q P C R)$. Total RNA was extracted from each experimental group using TRIzol ${ }^{\circledR}$ reagent (Thermo Fisher Scientific, Inc.), according to the manufacturer's protocol. qPCR for miR-370 was performed using miRNA primers obtained from Guangzhou RiboBio Co., Ltd. (Guangzhou, China). U6 was used as the internal control. The sequences were not supplied due to the rules of the company. The qPCR conditions were as follows: Denaturation at $94^{\circ} \mathrm{C}$ for $2 \mathrm{~min}$, amplifcation for 30 cycles at $94^{\circ} \mathrm{C}$ for $0.5 \mathrm{~min}$, annealing at $58^{\circ} \mathrm{C}$ for $0.5 \mathrm{~min}$ and extension at $72^{\circ} \mathrm{C}$ for $1 \mathrm{~min}$, followed by a terminal elongation step at $72^{\circ} \mathrm{C}$ for $10 \mathrm{~min}$. The RT-qPCR analysis was performed on a Bio-Rad CFX96 thermal cycler (Bio-Rad Laboratories, Inc., Hercules, CA, USA). Data was analyzed with the $2^{-\Delta \Delta C t}$ method. The same experiments were performed three times. GAPDH was used as the internal control.

Apoptosis analysis. Cells transfected with miR-NC or miR-370 were harvested at $48 \mathrm{~h}$ after transfection and stained with Annexin V-FITC/PI Apoptosis Detection kit (Keygentec, Nanjing, China) as the the manufacturer's protocol indicated. Apoptotic cells were assessed by flow cytometry (FACS Calibur; Becton Dickinson, Franklin Lakes, NJ, USA). The same experiments were performed three times.

The generation of stable clones. The SW620 cells were plated in 6 -well plate $\left(2 \times 10^{5}\right.$ cells/well). Then, the plasmid-based miR-370 expression system and miR-NC were transfected into cells using FuGENE HP ${ }^{\circledR}$ (Roche Diagnostics). After transfection for 48 h, 2 mg/ml G418 (Sigma-Aldrich, St. Louis, MO,
USA) was added into the medium and lasted for 10 days. The miR-370 expression in miR-370 and miR-NC cells were determined by qPCR. The survived cells was named as miR-370 cells (stable expression miR-370) and miR-NC cells.

Xenograft tumors in nude mice. Procedures involving animals conformed to the guidelines of the Institutional Animal Care and Use Committee of Sichuan Academy of Medical Sciences and Sichuan Provincial People's Hospital (Chengdu, China). SW620 cells stable expression miR-NC or miR-370 $\left(5 \times 10^{6}\right.$ cells in $100 \mu 1$ of DMEM) were injected subcutaneously into the flanks of nude mice (5 weeks old, male; Vital River Laboratories, Beijing China). Tumor volumes were measured at 10 days post cell injection and every five days. Tumor weights were measured immediately after sacrificing and tumor samples were harvested for RNA extraction and embedding in paraffin for TUNEL assay analysis.

TUNEL assay. For apoptotic cell detection in tumor tissues, serial $4-\mu \mathrm{m}$-thick tissue sections were prepared and dewaxed to water. Then DeadEnd ${ }^{\mathrm{TM}}$ Fluorometric TUNEL system (Promega Corporation, Madison, WI, USA) was performed according to the manufacturer's and the apoptotic cells is end-labeled with fluorophore. DAPI (Beyotime, Beijing, China) was purchased to stain cell nuclei. Fluorescence images were captured using a fluorescence microscopy (Olympus Corporation, Tokyo, Japan). The apoptotic index (apoptotic cells of total cell were analyzed. The same experiments were performed three times.

Luciferase assay. The precursor to miR-370 was synthesized and cloned in pMiRluc (Ambion Life Technologies, Carlsbad, CA, USA) to generate pMiRluc-370. Firefly luciferase reporter vectors with the intact putative miR-370 recognition sequence from the 3'-UTR of MDM4 or with random mutations cloned downstream of the firefly luciferase gene were constructed. For the 3'UTR-luciferase assays, 293T cells were co-transfected with MDM4-WT or mut-3'-UTR construct, pMiRluc or pMiRluc-miR-370 luciferase expression construct using FuGENE HP ${ }^{\circledR}$ (Roche Diagnostics). Luciferase assays were performed $24 \mathrm{~h}$ after transfection using the Dual Luciferase Reporter Assay system (Promega Corporation). Values were normalized to the activity of $\beta$-galactosidase.

Western blotting. The proteins were extracted in ice-cold RIPA lysis buffer (Beyotime) containing 1\% protease inhibitor cocktail (Pierce, Rockford, IL, USA), then centrifuged at $12,000 \mathrm{rpm}$ for $20 \mathrm{~min}$ at $4^{\circ} \mathrm{C}$. After protein concentration measurement by BCA assay kit (Beyotime), equivalent amounts of proteins were loaded on SDS-PAGE and transferred to PVDF membranes (Millipore, Bedford, MA, USA). After blocking by $5 \%$ non-fat milk in TBS/T buffer, antibodies against MDM4 (cat. no. 04-1555, 1:2,000; Millipore), p53 (cat. no. 2527, 1:1,000; Cell Signaling Technology, Inc., Danvers, MA, USA), $\gamma \mathrm{H} 2 \mathrm{AX}$ (cat. no. 9718, 1:1,000; Cell Signaling Technology, Inc.) and caspase 3 (cat. no. 9662, 1:1,000; Cell Signaling Technology, Inc.) were added for detection and all blots were probed with antibodies against $\beta$-actin (cat. no. 04-116, 1:3,000; Millipore) as loading control. ECL kit from Millipore was purchased for band exposure. The density of each band were measured by Image J software (National Institute of Health, Bethesda, MD, USA). 
Table I. Analysis of the correlation between miR-370 expression and clinicopathological characteristics.

\begin{tabular}{|c|c|c|c|c|}
\hline \multirow[b]{2}{*}{ Characteristics } & \multirow[b]{2}{*}{ Number of patients } & \multicolumn{2}{|c|}{ miR-370 expression } & \multirow[b]{2}{*}{ P-value } \\
\hline & & Low & High & \\
\hline $\operatorname{Sex}(\%)$ & & & & $>0.05$ \\
\hline Male & $13(43.3)$ & $5(38.5)$ & $8(61.5)$ & \\
\hline Female & $17(56.7)$ & $9(52.9)$ & $8(47.1)$ & \\
\hline Age $(\%)$ & & & & $>0.05$ \\
\hline$\leq 60$ & $10(33.3)$ & $4(40)$ & $6(60)$ & \\
\hline$>60$ & $20(66.7)$ & $6(50)$ & $6(50)$ & \\
\hline TNM stage $(\%)$ & & & & $<0.05$ \\
\hline I & $8(26.7)$ & $3(37.5)$ & $5(62.5)$ & \\
\hline II-III & $12(40)$ & $4(33.3)$ & $8(66.7)$ & \\
\hline IV & $10(33.3)$ & $2(20)$ & $8(80)$ & \\
\hline
\end{tabular}

TNM, tumor node metastasis; miR, microRNA.
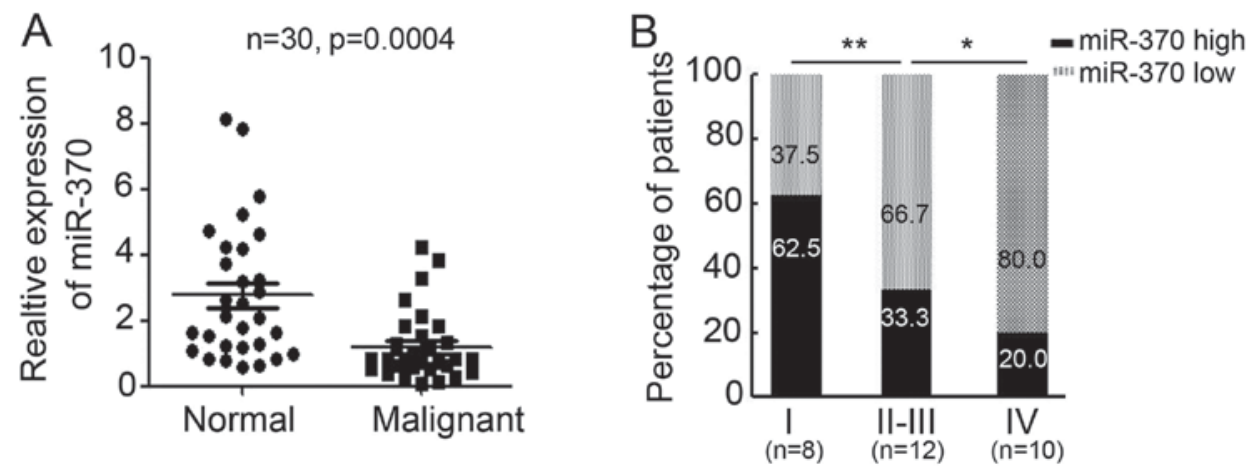

Figure 1. Expression of miR-370 in colonic malignant tissues. (A) Relative expression of miR-370 in 30 pairs of normal colon tissues and colonic malignant tissues, $n=30, P=0.0004$. GAPDH was used as the internal control. (B) Percentage of patients with high and low expression of miR-370 according to tumor grade, ${ }^{*} \mathrm{P}<0.05 ;{ }^{* *} \mathrm{P}<0.01$. miR, microRNA.

Immunohistochemical staining. SW620 xenograft tumors were instilled with $4 \%$ formalin and immersed in the same solution before tissue processing into paraffin-embedded blocks; $4 \mu \mathrm{m}$ sections were then cut for immunohistochemical staining. After antigen retrieval for 3 mins in citrate under high pressure, tumor tissue sections were incubated with primary antibody against MDM4 (cat. no. 04-1555, 1:300; Millipore) for immunohistochemical staining following the instructions of IHC kit (SP9001; ZsBio, Beijing, China) and DAB kit (Fuzhou Maixin Biotech Co., Ltd., Fuzhou, China). The stained tumors were visualized by a light microscope (DTX500; Nikon Corporation, Tokyo, Japan) The MDM4 positive cells were scored by counting the number of tumor cells expressing the proteins as determined by MDM4 staining in 5 random selected fields.

Statistical analysis. Statistical comparisons of all results were analyzed using analysis of variance (ANOVA) or t-test and represented graphically as mean \pm standard deviation (SD). All statistical analyses were carried out using SPSS 20.0 statistical software (SPSS, Inc., Chicago, IL, USA). P<0.05 was regarded as statistically significant.

\section{Results}

miR-370 expression is low in malignant colon tissue. To determine the expression of miR-370 in normal and malignant colon tissue, 30 pairs of colon tissues from patients were collected for qPCR testing (Table I). Interestingly, miR-370 expression levels were significantly lower in malignant colon tissues than in normal colon tissues (relative miR-370 expression in normal colon tissues, 2.772 \pm 0.3749 ; relative miR-370 expression in colon malignant tissues, 1.199 $\pm 0.1948 ; \mathrm{P}=0.0004$; Fig. 1A). Further analysis indicated miR-370 expression in grade I tumors was higher than in grade II-III or grade IV tumors (Fig. 1B). These results suggest that miR-370 is downregulated in malignant colon tissues and is inversely correlated with tumor grade.

Overexpression of miR-370 promotes apoptosis in colon cancer cells in vitro. Since miR-370 expression was reduced in malignant colon tissues, we were interested in its expression and role in colon cancer cells. We determined the expression level of miR-370 in several colon cancer cell lines and in normal human colon epithelial cells (HCoEpiC) and found miR-370 expression is significantly downregulated in colon 

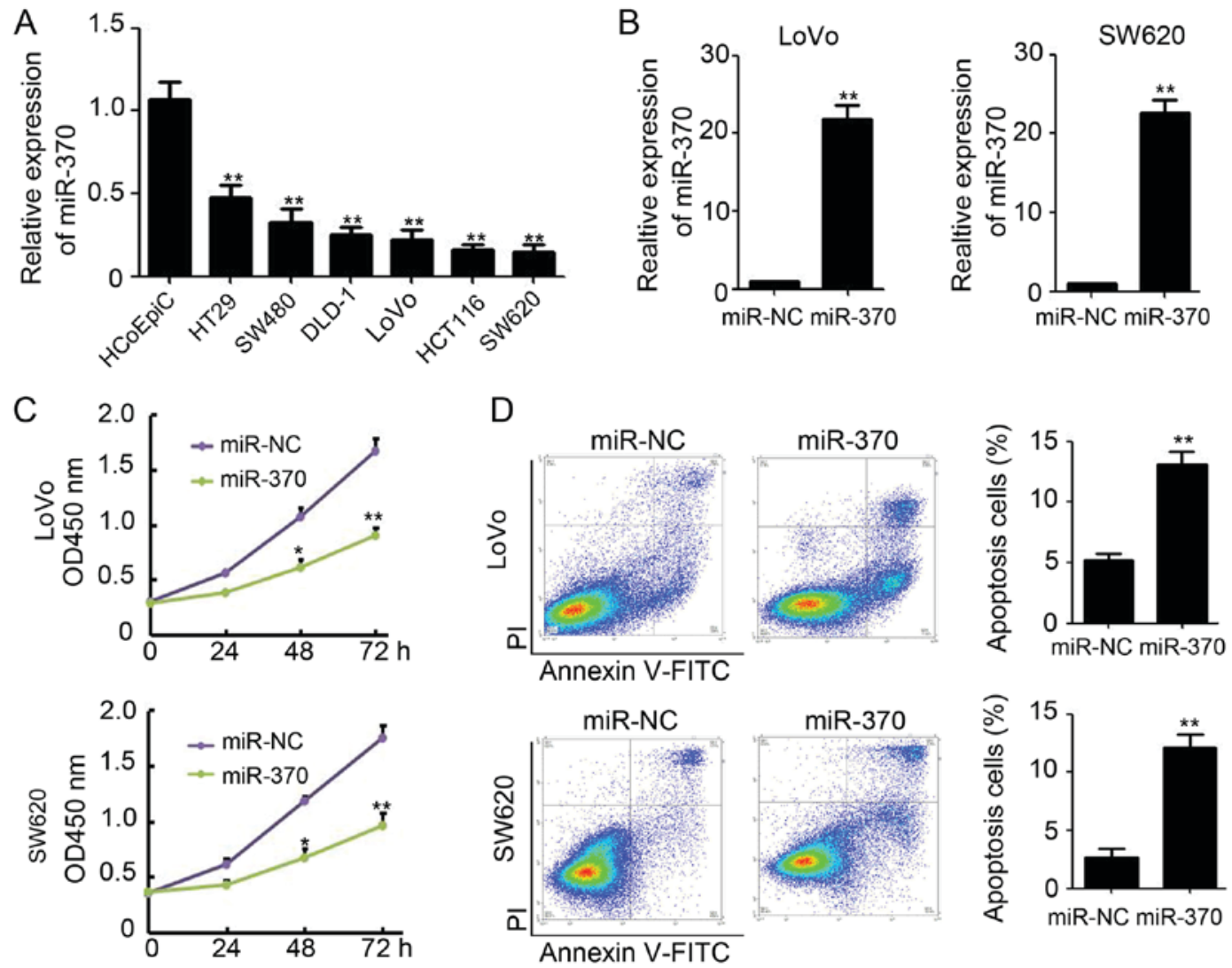

Figure 2. Overexpression of miR-370 promotes apoptosis in colon cancer cells in vitro. (A) qPCR analysis of miR-370 expression in HCoEpiCs and several colon cancer cells, $\mathrm{n}=3$; ${ }^{* *} \mathrm{P}<0.01$, compared with HCoEpiCs. GAPDH was used as the internal control. (B) $\mathrm{qPCR}$ analysis of miR-370 expression in miR-NC and miR-370 transfected LoVo and SW620 cells, $n=3 ;{ }^{* * *} \mathrm{P}<0.01$, compared with miR-NC transfected cells. GAPDH was used as the internal control. (C) Analysis of cell viability by CCK-8 in miR-NC and miR-370 transfected LoVo and SW620 cells, $\mathrm{n}=3$; ${ }^{*} \mathrm{P}<0.05 ;{ }^{* * *} \mathrm{P}<0.01$, compared with miR-NC transfected cells. (D) Analysis of cell apoptosis by Annexin V and PI in miR-NC and miR-370 transfected LoVo and SW620 cells, n=3; ${ }^{* *} \mathrm{P}<0.01$, compared with miR-NC transfected cells. miR, microRNA; qPCR, quantitative real-time polymerase chain reaction; CCK-8, Cell Counting kit-8.

cancer cells (Fig. 2A). LoVo and SW620, which get lowest miR-370 expression among the investigated colon cancer cell lines, were selected for further research. Next, we introduced a plasmid-based miR-370 expression system and demonstrated that the plasmid effectively enhanced miR-370 levels in colon cancer cell lines (LoVo and SW620) (Fig. 2B). Overexpression of miR-370 in LoVo and SW620 cells resulted in significantly reduced viability compared to the miR-NC (negative control) transfected group. Viability was reduced by $52.5 \%$ in LoVo cells and 56.6\% in SW620 cells (Fig. 2C). Furthermore, an improved rate of apoptosis in LoVo and SW620 cells was observed upon treatment with miR-370 (LoVo, $5.2 \pm 0.5 \%$ for miR-NC vs. $13.1 \pm 1.1 \%$ for miR-370, $\mathrm{P}<0.01$; SW620, $2.7 \pm 0.7 \%$ for miR-NC vs. $12.7 \pm 1.6 \%$ for miR-370, P<0.01; Fig. 2D). These results indicate that overexpression of miR-370 inhibits colon cell proliferation through apoptotic pathways, in vitro.

Overexpression of miR-370 reduces colon tumor growth in vivo. To validate the observed phenomenon in vivo, SW620 cells that stably express either miR-NC or miR-370 were used to establish xenograft tumor models in nude mice. $\mathrm{qPCR}$ results showed that the expression of miR-370 in miR-370 stably transfected SW620 cells was higher than in miR-NC stable transfected SW620 cells (Fig. 3A). We found that overexpression of miR-370 in colon cancer cells markedly reduced xenograft tumor growth (Fig. 3B). Tumor volume decreased by $63.6 \%\left(1,872.3 \pm 283.8 \mathrm{~mm}^{3}\right.$ for
miR-NC vs. $682.3 \pm 83.4 \mathrm{~mm}^{3}$ for miR-370; $\mathrm{P}<0.01$; Fig. $3 \mathrm{C}$ ) and tumor weight decreased by $64.9 \%(1.69 \pm 0.13 \mathrm{~g}$ for miR-NC vs. $0.61 \pm 0.15 \mathrm{~g}$ for miR-370; $\mathrm{P}<0.01$; Fig. 3D). Furthermore, TUNEL staining suggested a significantly increased apoptotic index upon transfection of SW620 xenograft tumors with miR-370 (Fig. 3E). These results indicate that overexpression of miR-370 retards colon tumor growth in vivo.

miR-370 directly targets MDM4 to promote apoptosis in colon cancer cells. To investigate the possible mechanisms by which miR-370 promotes apoptosis in colon cancer cells, TargetScan and miRDB were used to identify miR-370's predicted biological target, MDM4. To verify this analysis, the MDM4 3'UTR, containing the miR-370 binding site, was cloned downstream of the luciferase open reading frame. Additionally, a MDM4 3'UTR mutant, which contains a mutated miR-370 binding site, was also introduced into the luciferase construct (Fig. 4A). Next, 293T cells, which were widely used as a tool cell in luciferase assay (14), were performed to investigate whether miR-370 directly targets MDM4. qPCR results showed that the expression of miR-370 in miR-370 transfected 293T cells was higher than in miR-NC transfected $293 \mathrm{~T}$ cells (Fig. 4B). After the luciferase-MDM4-3'UTR transfection, luciferase expression in miR-370 transfected $293 \mathrm{~T}$ cells was significantly affected (Fig. 4C). Results showed consistent reduction of luciferase expression in miR-NC and miR-370 transfected 293T cells 
A

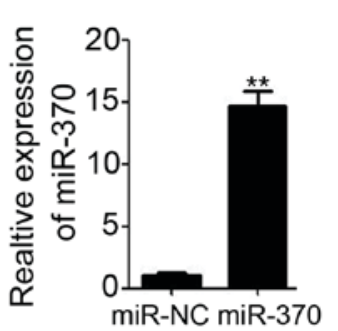

B

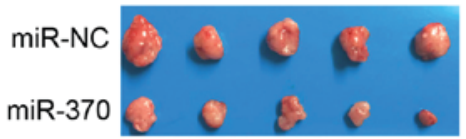

C

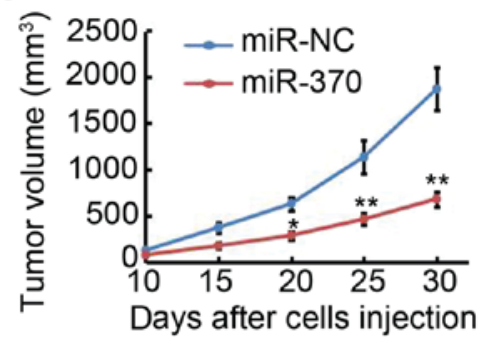

D

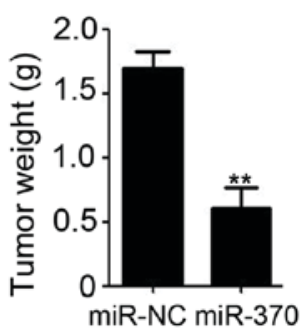

E

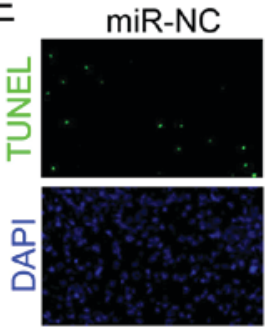

miR-370

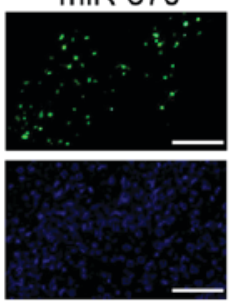

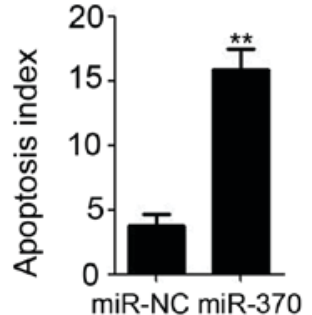

Figure 3. Overexpression of miR-370 reduces colon tumor growth in vivo. (A) qPCR analysis of miR-370 expression in SW620 cells that stably transfected with miR-370 and miR-NC, $n=3 ;{ }^{* * *} \mathrm{P}<0.01$, compared with miR-NC cells. (B) Representative macroscopic findings of colon tumors. (C) Tumor volume after injection of $5 \times 10^{6}$ miR-NC or miR-370 SW620 cells into nude mice. $\mathrm{n}=5 ;{ }^{*} \mathrm{P}<0.05 ;{ }^{* * *} \mathrm{P}<0.01$, compared with miR-NC group. (D) End-stage weight after injection of $5 \times 10^{6}$ miR-NC or miR-370 SW620 cells into nude mice. $\mathrm{n}=5$; ${ }^{* *} \mathrm{P}<0.01$, compared with miR-NC group. (E) TUNEL analysis of apoptosis cells in miR-NC and miR-370 xenograft tumors, $\mathrm{n}=3 ;{ }^{* * *} \mathrm{P}<0.01$, compared with miR-NC group. miR, microRNA; qPCR, quantitative real-time polymerase chain reaction.

A

5'AgCAACUUUAUGAUCAGCAGGA-3' MDM4-3'UTR
I| ||$!|||||| \mid$ 3'UGGUCCAAGGUGGGGUCGUCCG-5' miR-370 5'AgCAACUUUAUGAUGUCGAGGA3' MDM4-3'UtR mutant
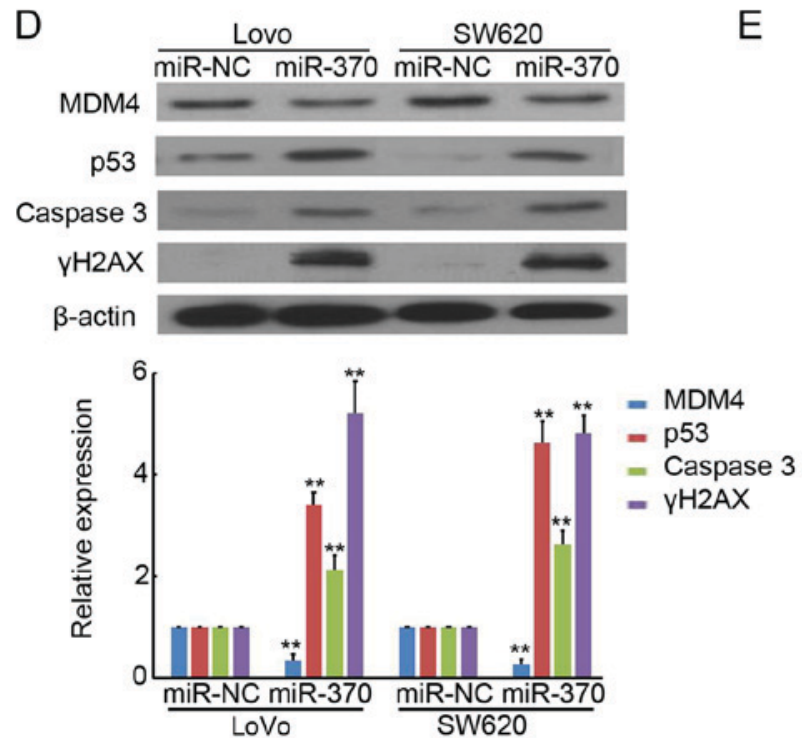

B

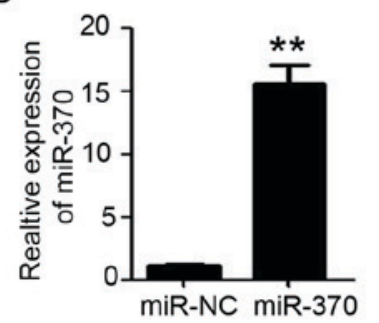

E

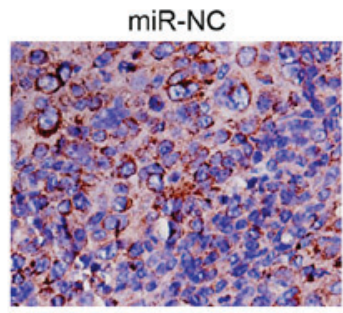

C
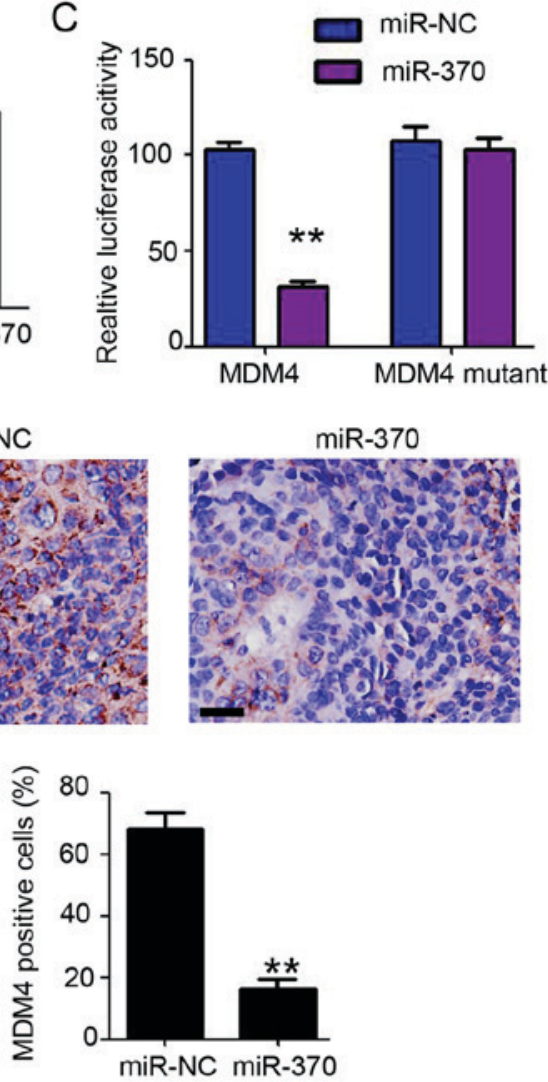

Figure 4. miR-370 directly targets MDM4. (A) MDM4-3'UTR contains one predicted miR-370 binding site. The figure shows predicted duplex formations between MDM4-3'UTR and miR-370. The sites of target mutagenesis are indicated in blue. (B) qPCR of miR-370 in 293T cells after enforced expression of miR-370 in 293T cells. ${ }^{* *} \mathrm{P}<0.01$, compared to miR-NC group. (C) Relative repression of luciferase expression was standardized to a transfection control. ${ }^{* *} \mathrm{P}<0.01 \mathrm{compared}$ to miR-NC control. (D) Western blot analysis of MDM4, p53, caspase 3 and $\gamma \mathrm{H} 2 \mathrm{AX}$ expression in miR-NC and miR-370 transfected LoVo and SW620 cells. $\beta$-actin was used as loading control. Relative expression analysis of MDM4, p53, caspase 3 and $\gamma \mathrm{H} 2 \mathrm{AX}$ expression. $\mathrm{n}=3,{ }^{* * *} \mathrm{P}<0.01$, compared with miR-NC transfected group. (E) IHC staining of MDM4 in miR-NC and miR-370 xenograft tumor tissues. Scale bar, $50 \mu \mathrm{m}$. The percentage of MDM4 positive cells were counted and analyzed. $n=5,{ }^{* *} \mathrm{P}<0.01$, compared with miR-NC group. miR, microRNA; MDM4, mouse double minute 4; qPCR, quantitative real-time polymerase chain reaction. 
A

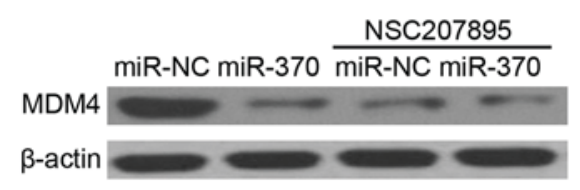

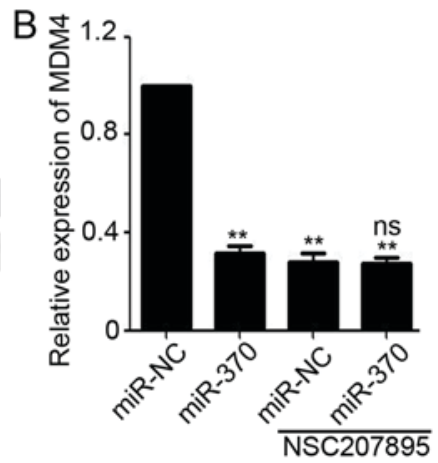

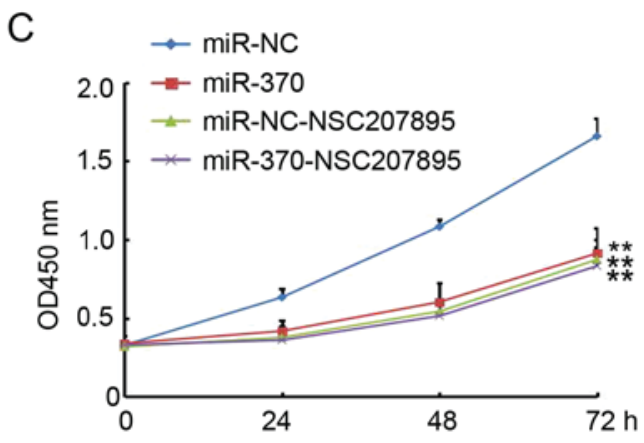

Figure 5. MDM4 plays a necessary role during miR-370-mediated cancer cell growth inhibition. (A) Western blotting determination of MDM4 expression in miR-NC and miR-370 transfected LoVo cells treated with or without NSC207895. $\beta$-actin was used as loading control. (B) Relative expression analysis of MDM4 expression. $\mathrm{n}=3,{ }^{* *} \mathrm{P}<0.01$, compared with miR-NC transfected group; $\mathrm{ns}$, no significant difference, compared with miR-NC transfected group with NSC207895 treatment. (C) Analysis of cell viability by CCK-8 kit in miR-NC and miR-370 transfected LoVo cells that treated with or without NSC207895. ${ }^{* *} \mathrm{P}<0.01$, compared with miR-NC group. MDM4, mouse double minute 4; miR, microRNA; CCK-8, Cell Counting kit-8.

following transfection with miRNA binding site mutant plasmids (luciferase-MDM4-3'UTR mutant) (Fig. 4C). Further western blot analysis demonstrated that miR-370 reduced MDM4 expression in LoVo and SW620 cells, accompanied by increased p53, caspase 3 and $\gamma \mathrm{H} 2 \mathrm{AX}$ expression, both of which are downstream targets of MDM4 (Fig. 4D). IHC staining also demonstrated less MDM4 positive cells were found upon transfection of SW620 xenograft tumors with miR-370, compared with miR-NC group (Fig. 4E). These results suggest that miR-370 directly targets MDM4 to promote apoptosis in colon cancer cells.

MDM4 plays a necessary role during miR-370-mediated cancer cell growth inhibition. Next, to investigate whether MDM4 plays a necessary role during miR-370-mediated cancer cell growth inhibition, MDM4 specific inhibitor NSC207895 was purchased and used to treat miR-370 or miR-NC transfected LoVo cells. As shown in Fig. 5A and B, NSC207895 efficiently inhibited the expression of MDM4 in miR-NC transfected LoVo cells. Meanwhile, there was no significant difference on MDM4 expression in miR-NC and miR-370 transfected cells that after NSC207895 treatment (Fig. 5A and B). Furthermore, CCK-8 assay demonstrated that miR-370 transfected resulted in significantly reduced viability compared to the miR-NC transfected LoVo cells (Fig. 5C). But NSC207895 efficiently blocked the miR-370-mediated Loco cell growth inhibition (Fig. 5C). Collectively, these results indicated that MDM4 plays a necessary role during miR-370-mediated cancer cell growth inhibition.

\section{Discussion}

In the present study, we investigated the expression patterns and role of miR-370 in colon cancer cells. We discovered downregulation of miR-370 expression in malignant colon tissue and miR-370 expression was inversely correlated with tumor grade. Moreover, we determined that miR-370 functions as a tumor suppressor in colon cancer by inhibiting tumor growth and promoting cell apoptosis. Mechanistically, MDM4 was demonstrated to be a potential direct target of miR-370 during colon cancer cell apoptosis. Collectively, these findings suggest that upregulation of miR-370 may impair colon tumor growth by targeting MDM4.
Recently, case series studies demonstrated the expression and function of miR-370 in cancer. Previous studies showed that miR-370 is decreased in the tissues and cell lines of gastric cancer and overexpression of miR-370 promotes cell apoptosis and inhibits proliferation $(15,16)$. In non-small cell lung cancer tissues and cell lines, miR-370 expression was also downregulated, and upregulation of miR-370 impaired tumor growth by inhibiting cell proliferation (17). Meng et al have investigated the mechanism of miR-370 downregulation; overexpression of IL-6 induced epigenetic-mediated miR-370 downregulation in malignant human cholangiocytes (18). However, the expression and function of miR-370 in colon cancer remains unclear. In the present study, we reported that miR-370 was downregulated in colon cancer tissues compared to the adjacent tissues of the tumor, and confirmed miR-370 downregulation in colon cancer cell lines. Furthermore, miR-370 expression inversely correlated with tumor grade. Importantly, we identified the tumor suppressor role of miR-370 in colon cancer. Overexpression of miR-370 impairs the xenograft tumor growth and cell proliferation in vivo and in vitro by promoting colon cancer cell apoptosis. These results provide solid evidence for understanding the role of miR-370 in colon cancer. Regrettably, we didn't examine the expression of miR-370 in clinic samples after effective treatment. And further research is required to investigate the expression of miR-370 following treatment.

MDM4, a master regulator of p53, binds its homologue MDM2 and this results in p53 activity repression through MDM2-driven ubiquitination (19). As a cytoplasmic protein, MDM4 negatively controls p53 transcriptional activity (20). Therefore,MDM4 and the MDM2-MDM4 complex are the key regulators of p53 protein stability and are involved in p53 activation (21). In colon cancer, MDM4 was detected in approximately $50 \%$ of human colon tumors and showed strong correlation with increased extracellular signal-regulated kinase phosphorylation (22). MDM4 expression in colon cancer was regulated by several factors. FL118 promotes degradation of MDM4, leading to p53-dependent apoptosis (23). Furthermore, DNA damage-induced miR-34a overexpression decreases MDM4 protein levels in colon cancer, based on the presence of a miR-34a binding site (24). MDM4 expression is also regulated by mitogenic signaling pathways (22) and genotoxic stress (25). In the present study, luciferase assays and western 
blot analysis revealed that MDM4 expression was downregulated by miR-370. Through an 11 mer binding site, the MDM4 3'-UTR is a target of miR-370. Overexpression of miR-370 in colon cancer cells inhibits MDM4 protein expression and promotes $\mathrm{p} 53$, caspase 3 and $\gamma \mathrm{H} 2 \mathrm{AX}$ expression, resulting in cell apoptosis. Notably, as shown in Fig. 2C and D, only $15 \%$ apoptotic cells resulted in a 50-60\% reduction in viability/death. It may contributed to that the reduction in cell viability was a accumulated effect of apoptosis, that was persistent after miR-370 transfection. Furthermore, MDM4 expression in SW620 xenograft tumor tissues was also inhibited by overexpression of miR-370. Previous studies by Li et al (26) and Pan et al (27) have demonstrated that miR-370 can activated p21 expression in lung cancer cells and bladder cells and inhibit PIM1 expression in hepatocellular carcinoma. But in our study, inhibition of MDM4 by specific inhibitor NSC207895 efficiently blocked miR-370-mediated cell growth inhibition (Fig. 5). It's demonstrated that MDM4 plays a necessary role during miR-370 inhibiting colon cancer cell growth. The diversity of mechanism under miR-370 inhibiting cancer cell growth would contributed to the different cancer cells we investigated. But whether the miR-370-mediated induction of apoptotic cell death in colon cancer cells is p53 dependent should be investigated in the further study. We also need determine the expression of MDM4 in clinic colon tumors and analyze the correlation between miR-370 and MDM4 expression in clinic samples. It may provide solid evidence for understanding the regulation role of miR-370 on MDM4 expression.

In conclusion, our research demonstrated that there is downregulation of miR-370 in malignant colon tissue and its level is inversely correlated with tumor grade. Functional studies found that miR-370 directly targets the oncogene MDM4 and inhibits the growth of colon cancer in vitro and in vivo. Taken together, our data provide clear evidence that miR-370 functions as a tumor suppressor and may serve as an effective target for the treatment of colon cancer.

\section{Acknowledgements}

The present study was supported by Doctoral Fund of Sichuan Provincial People's Hospital (no. 30305030584).

\section{References}

1. Siegel RL, Miller KD and Jemal A: Cancer statistics, 2017. CA Cancer J Clin 67: 7-30, 2017.

2. Calvert PM and Frucht H: The genetics of colorectal cancer. Ann Intern Med 137: 603-612, 2002.

3. Wei EK, Wolin KY and Colditz GA: Time course of risk factors in cancer etiology and progression. J Clin Oncol 28: 4052-4057, 2010.

4. Dai L, Cui X, Zhang X, Cheng L, Liu Y, Yang Y, Fan P, Wang Q, Lin Y, Zhang $\mathrm{J}$ et al: SARI inhibits angiogenesis and tumour growth of human colon cancer through directly targeting ceruloplasmin. Nat Commun 7: 11996, 2016.

5. Chawla JP, Iyer N, Soodan KS, Sharma A, Khurana SK and Priyadarshni P: Role of miRNA in cancer diagnosis, prognosis, therapy and regulation of its expression by Epstein-Barr virus and human papillomaviruses: With special reference to oral cancer. Oral Oncol 51: 731-737, 2015.

6. Gambari R, Brognara E, Spandidos DA and Fabbri E: Targeting oncomiRNAs and mimicking tumor suppressor miRNAs: Nuew trends in the development of miRNA therapeutic strategies in oncology (Review). Int J Oncol 49: 5-32, 2016.
7. Li Y, Xu Z, Li B, Zhang Z, Luo H, Wang Y, Lu Z and Wu X: Epigenetic silencing of miRNA-9 is correlated with promoter-proximal $\mathrm{CpG}$ island hypermethylation in gastric cancer in vitro and in vivo. Int J Oncol 45: 2576-2586, 2014.

8. Lv J, Xia K, Xu P, Sun E, Ma J, Gao S, Zhou Q, Zhang M, Wang F, Chen F, et al: miRNA expression patterns in chemoresistant breast cancer tissues. Biomed Pharmacother 68: 935-942, 2014.

9. Molina-Pinelo S, Carnero A, Rivera F, Estevez-Garcia P, Bozada JM, Limon ML, Benavent M, Gomez J, Pastor MD, Chaves M, et al: MiR-107 and miR-99a-3p predict chemotherapy response in patients with advanced colorectal cancer. BMC Cancer 14: 656, 2014.

10. Nadal E, Zhong J, Lin J, Reddy RM, Ramnath N, Orringer MB, Chang AC, Beer DG and Chen G: A MicroRNA cluster at 14q32 drives aggressive lung adenocarcinoma. Clin Cancer Res 20: 3107-3117, 2014

11. Yungang W, Xiaoyu L, Pang T, Wenming L and Pan X: miR-370 targeted FoxM1 functions as a tumor suppressor in laryngeal squamous cell carcinoma (LSCC). Biomed Pharmacother 68: 149-154, 2014.

12. Chen XP, Chen YG, Lan JY and Shen ZJ: MicroRNA-370 suppresses proliferation and promotes endometrioid ovarian cancer chemosensitivity to cDDP by negatively regulating ENG. Cancer Lett 353: 201-210, 2014

13. Wu Z, Sun H, Zeng W, He J and Mao X: Upregulation of MircoRNA-370 induces proliferation in human prostate cancer cells by downregulating the transcription factor FOXO1. PLoS One 7: e45825, 2012.

14. Luo P, He T, Jiang R and Li G: MicroRNA-423-5p targets O-GlcNAc transferase to induce apoptosis in cardiomyocytes. Mol Med Rep 12: 1163-1168, 2015.

15. Zeng Y, Fu M, Wu GW, Zhang AZ, Chen JP, Lin HY, Fu YA, Jia J, Cai ZD, Wu XJ and Lan P: Upregulation of microRNA-370 promotes cell apoptosis and inhibits proliferation by targeting PTEN in human gastric cancer. Int J Oncol 49: 1589-1599, 2016.

16. Lo SS, Hung PS, Chen JH, Tu HF, Fang WL, Chen CY, Chen WT, Gong NR and Wu CW: Overexpression of miR-370 and downregulation of its novel target TGFbeta-RII contribute to the progression of gastric carcinoma. Oncogene 31: 226-237, 2012.

17. Chen T, Gao F, Feng S, Yang T and Chen M: MicroRNA-370 inhibits the progression of non-small cell lung cancer by downregulating oncogene TRAF4. Oncol Rep 34: 461-468, 2015.

18. Meng F, Wehbe-Janek H, Henson R, Smith H and Patel T: Epigenetic regulation of microRNA-370 by interleukin- 6 in malignant human cholangiocytes. Oncogene 27: 378-386, 2008.

19. Hirose M, Yamato K, Endo S, Saito R, Ueno T, Hirai S, Suzuki H, Abei M, Natori Y and Hyodo I: MDM4 expression as an indicator of TP53 reactivation by combined targeting of MDM2 and MDM4 in cancer cells without TP53 mutation. Oncoscience 1: 830-843, 2014.

20. Tan BX, Khoo KH, Lim TM and Lane DP: High Mdm4 levels suppress p53 activity and enhance its half-life in acute myeloid leukaemia. Oncotarget 5: 933-943, 2014.

21. Wang MJ, Luo YJ, Shi ZY, Xu XL, Yao GL, Liu RP and Zhao H: The associations between MDM4 gene polymorphisms and cancer risk. Oncotarget 7: 55611-55623, 2016.

22. Gilkes DM, Pan Y, Coppola D, Yeatman T, Reuther GW and Chen J: Regulation of MDMX expression by mitogenic signaling. Mol Cell Biol 28: 1999-2010, 2008.

23. Ling X, Xu C, Fan C, Zhong K, Li F and Wang X: FL118 induces p53-dependent senescence in colorectal cancer cells by promoting degradation of MdmX. Cancer Res 74: 7487-7497, 2014.

24. Mandke P, Wyatt N, Fraser J, Bates B, Berberich SJ and Markey MP: MicroRNA-34a modulates MDM4 expression via a target site in the open reading frame. PLoS One 7: e42034, 2012.

25. Markey M and Berberich SJ: Full-length hdmX transcripts decrease following genotoxic stress. Oncogene 27: 6657-6666, 2008

26. Li C, Ge Q, Liu J, Zhang Q, Wang C, Cui K and Chen Z: Effects of miR-1236-3p and miR-370-5p on activation of p21 in various tumors and its inhibition on the growth of lung cancer cells. Tumour Biol 39: 1010428317710824, 2017.

27. Pan XP, Wang HX, Tong DM, Li Y, Huang LH and Wang C: miRNA-370 acts as a tumor suppressor via the downregulation of PIM1 in hepatocellular carcinoma. Eur Rev Med Pharmacol Sci 21: 1254-1263, 2017.

This work is licensed under a Creative Commons Attribution-NonCommercial-NoDerivatives 4.0 International (CC BY-NC-ND 4.0) License. 